\title{
EXPERT SYSTEM TO DIAGNOSE DENGUE FEVER
}

\author{
MOHD ZULHILMI AQIL MUHAMAD ${ }^{\mathrm{A}}$, AND NOOR HAFHIZAH ABD RAHIM ${ }^{\mathrm{B}, *}$ \\ ${ }^{a, b}$ Faculty of Ocean Engineering Technology and Informatics, Universiti Malaysia Terengganu, 21030 Kuala Nerus, \\ Terengganu, Malaysia
}

*Corresponding author: noorhafhizah@umt.edu.my

\begin{abstract}
Expert system is a system that emulates experts to aid in decision making. This system can be applied in various categories such as diagnosis, prediction, interpretation, and others. Expert System to Diagnose Dengue Fever is a web-based system which is integrated with prolog language in order to provide rules for dengue fever detection. The aims of this research are to study dengue fever symptoms and other illnesses related to the fever, to design an inference engine, and to build an expert system. The challenges faced while developing this expert system were the complexity of prolog codes and their integration with the web development. In this system, rules were developed by prolog language which define dengue fever and accuracy based on input from the user. This system is expected to aid users in self-detecting early symptoms of dengue fever before seeing the doctors.
\end{abstract}

Keywords: dengue fever, PROLOG, symptoms, expert system.

\section{Introduction}

Expert system is a system with human intelligence that is used to solve real-world problems. According to Sharifah Hanis Syed Ahmad (2012), expert system is a rule-based system, where results obtained from the rules are similar to human knowledge in specific areas. Expert system can be applied in many areas, such as industries, commercial problems, financial decision making, and diagnosis of diseases. In this study, expert system to diagnose dengue was developed.

Dengue is caused by a virus carried by Aedes mosquitos which can cause severe illness. If not treated as soon as possible, it can lead to death. This has been stated in a report from WHO, which mentioned that in most Asian and Latin American countries, dengue becomes the number one cause of death and hospitalization cases among kids and adults (Dengue and Severe Dengue, April 2017).

The history of dengue began in 1780 , when the first case occurred and subsequent cases spread in North America, Africa and Asia continents, with the disease not yet identifiable. However, in 1950s, the most severe dengue was identified, and the disease became epidemics in Thailand and the Philippines (Dengue and Severe Dengue, April 2017). Dengue transmission could only be identified in the 20th century. Approximately 2.5 billion or $40 \%$ of the current population is in a state of risk and may be susceptible to dengue (History of Dengue (n.d.)).

Dengue fever does not have specific method of treatment. Early detection and proper treatment can reduce the threat rate to $1 \%$. Dengue can be identified early through symptoms such as body aches and lack of appetite. These symptoms have been officially confirmed by the relevant parties before being broadcast in mass media such as television as a way to help the public identify and prevent the dengue epidemic from becoming more serious. The aim of the expert system which diagnoses dengue fever is to help the users of the system to determine whether they are suffering from the diseases or not. The system can provide diagnoses based on symptoms of dengue fever similar to the experts.

Dengue fever is a global epidemic that threatens humans. The dengue virus carried by Aedes mosquitoes has spread throughout Malaysia. Malaysians need to know about the 
epidemic and how to prevent and neutralize it. Statistics for the cases collected from 1 January 2017 until 8 November 2017 showed that Malaysia had about 76,247 cases all over the country (Bilik Gerakan Denggi Kebangsaan CPRC, Kementerian Kesihatan Malaysia (KKM), 2017). The real problem is people cannot identify whether they have contracted dengue fever or not before seeing a doctor.

The objectives of this research are to study the problem domain and other expert systems related to dengue fever and other illnesses, and to design and build an expert system that can identify dengue fever to help users diagnose themselves. The scope of this research was specific to dengue fever. The system's platform is web-based and suitable to be used by Malaysians. The expected outcome from this research was a system developed using PROLOG, which provides artificial intelligence that is able to give results based on symptom input from users, thereby providing a convenient way to get a diagnose for dengue fever instead of having to see a doctor.

\section{Literature Review}

This literature review describes research on previous systems and observations of what the previous systems could do in relation to diagnosing dengue. There have been several attempts to develop the prototypes of dengue expert systems, and these previous attempts by the researchers are described here. This literature review will recap five previous expert systems that had been developed, such as "Adaptive Neuro-Fuzzy Inference System for diagnosis risk in dengue patient" and "An Expert System for Diagnosis of Human Diseases".

\section{Adaptive Neuro-Fuzzy Inference System (ANFIS) for diagnosis risk in dengue patient}

This inference system was developed by Tarig Faisal et. al (2012) for diagnosis risk in dengue patients. The system applies Artificial Neural Network (ANN) concept to determine its characteristics in a way to develop the system. The system can be used during diagnoses of dengue patients' level of risk by physicians. This system was proposed to be used by physicians. This system is able to aid doctors in identifying the risk level of dengue patients. Then, they can make precise decision for their patients. This ANFIS system is integrated with two diagnostic models as illustrated in Figure 1. The first model was developed by systematically optimizing the initial ANFIS model parameters, while the second model was developed by employing the subtractive clustering algorithm to optimize the initial ANFIS model parameters. Figure 2 shows the model. Based on the experiments, the clustering technique integrated to ANFIS had better performance than the other model.

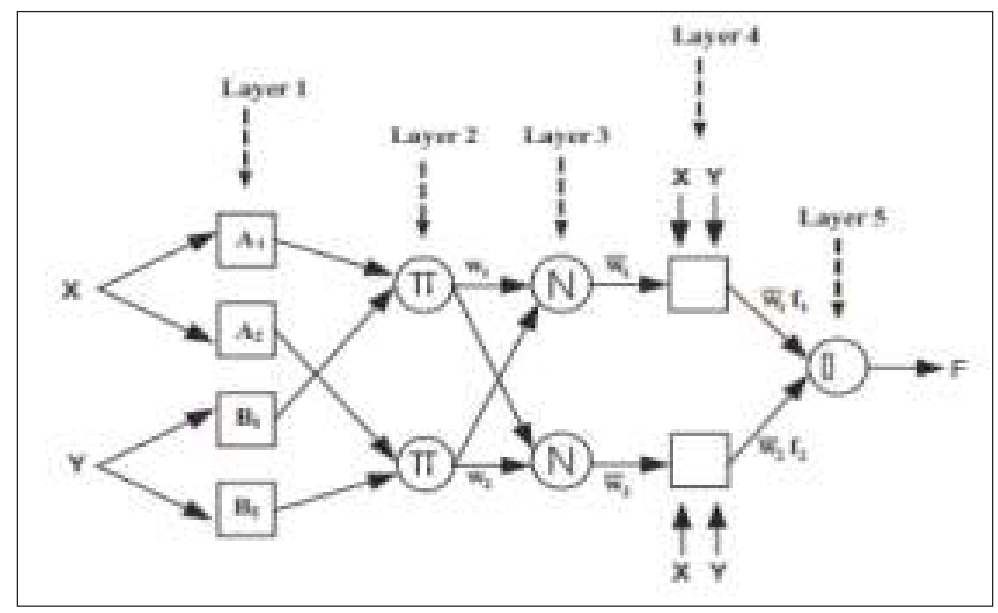

Figure 1: Architecture of ANFIS 


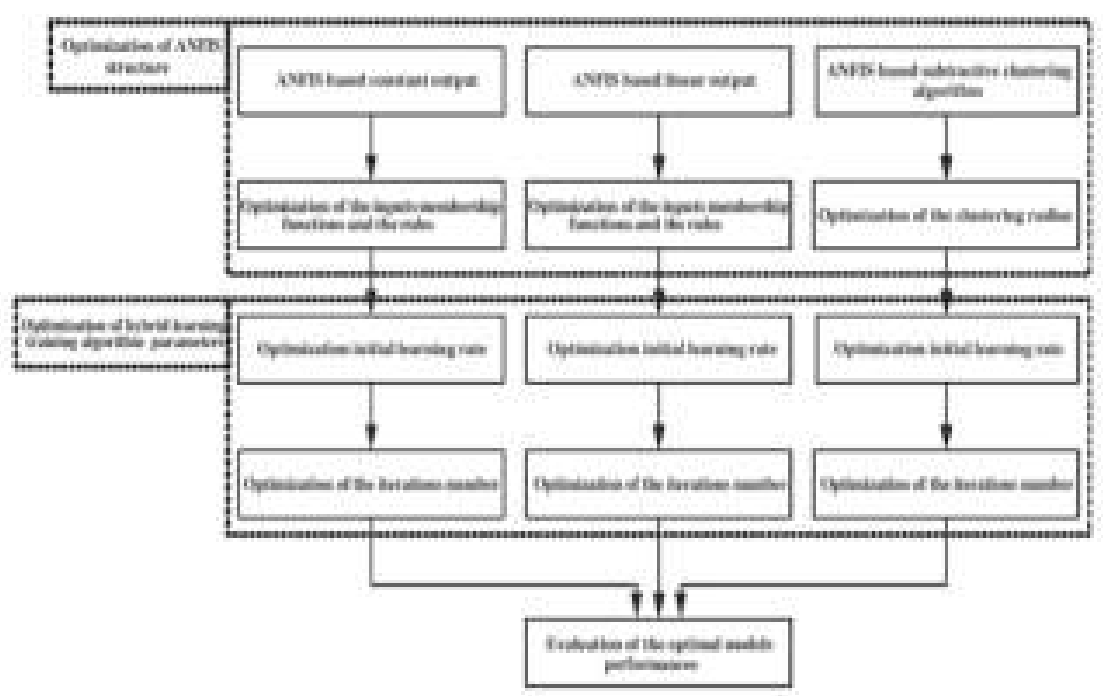

Figure 2: ANFIS model

\section{An Expert System for Diagnosis of Human} Diseases

Santosh Kumar Patra et. al (2010) developed an expert system to diagnose human diseases. The system applies rule-based technique and makes inferences with symbols. In order to understand the symbolic structure, it needs the translation from domain experts. The system can be used everywhere. The system was developed to detect diseases at an early stage so that people can get appropriate treatment immediately. There are a few phases in this system, as shown in Figure 3. In the first phase, physicians record medical records of their patients. This activity is done by conducting personal interviews with the patients. Next, a set of rules based on symptoms and diseases is developed. The rules are written based on IF and THEN structures. In order to fire the rules, forward reasoning was chosen in inference engine. This task aims to combine the facts and applicable rules from the rule database. In the final step, the diseases are produced in the inference engine. The symptoms for the diseases are identified by this expert system.

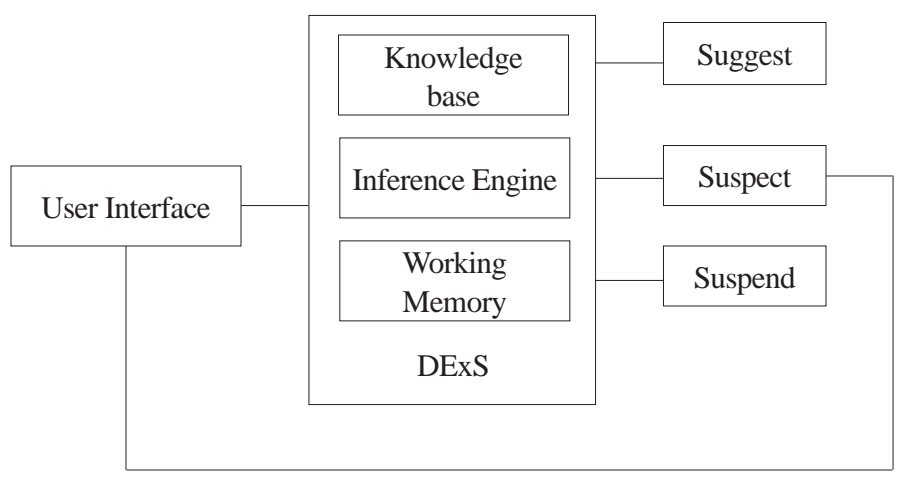

Figure 3: DExS working model 
An Expert System Oriented towards the Detection of Influenza and Dengue Developed on Mobile Platforms (BioDnX)

BioDnX was developed by Raúl Beltrán Ramírez et. al (2015). The system was developed using fuzzy logic as to deal with the uncertainty. This expert system can be used when a person is unwell and needs a diagnosis to identify whether he/she is infected by dengue or influenza. The system is an application that is mobile-based and available for anyone with a smartphone or tablet everywhere. The system was developed due to symptoms of sickness that can be confusing or other simple sicknesses which in many cases can lead to a late diagnosis. The expert system works by calculating every symptom, paying close attention to the slightest of details. The user selects the symptoms presented, and the system calculates the probability of the user having each disease. Finally, the diagnosis is determined based on the keyed-in symptoms. The flows are illustrated in Figure 4.

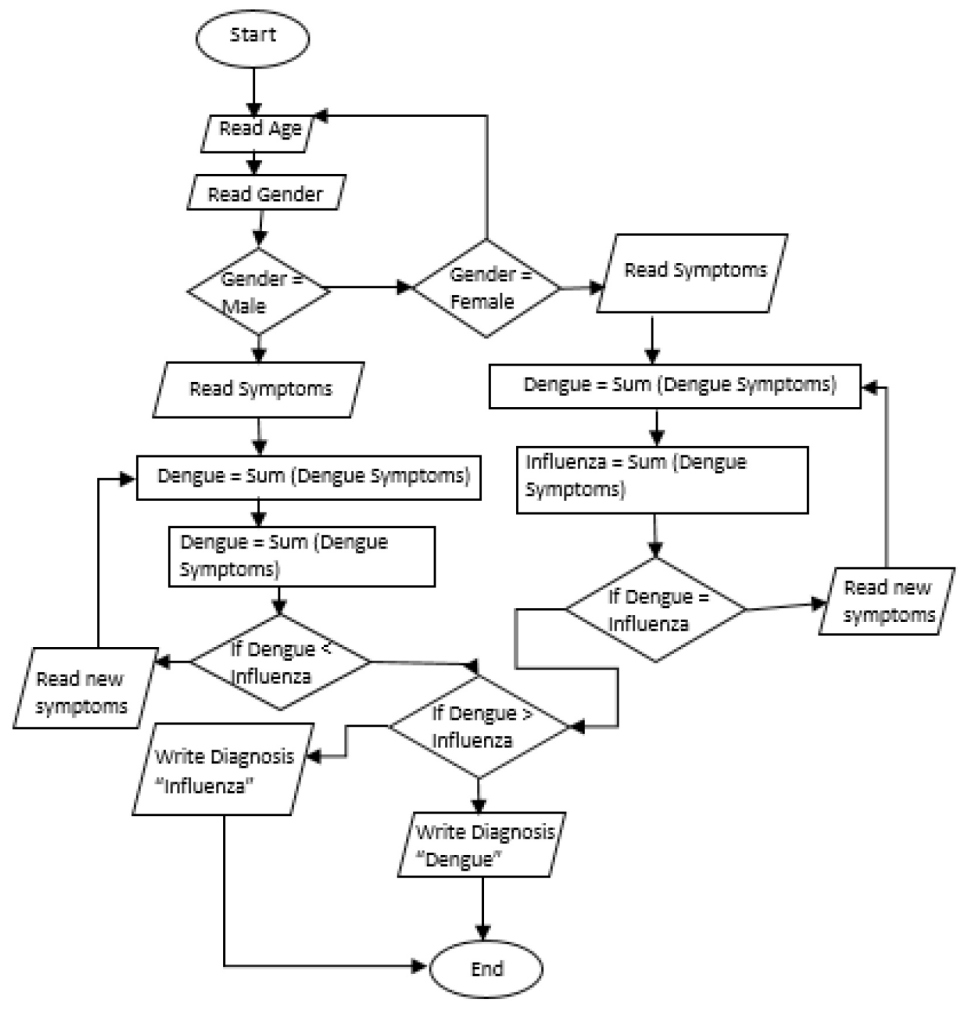

Figure 4: Flowchart of BioDnX

\section{A Belief Rule-Based Expert System to Diagnose Influenza}

This system was developed by Hossain et. al (2014). RIMER methodology was used to develop the system. The system is used to diagnose whether the patients have contracted influenza. The system can be used in any medical centres. The traditional system is unable to deliver desired accuracy. This expert system works by applying Evidential Reasoning (ER) algorithm that is used as an inference mechanism or to deduce inference in RIMER. BRB is the extended form of traditional IF-THEN rulebase that contains appropriate schema to capture different types of uncertainties and allows handling of non-linear causal relationships. 
Evidential Reasoning (ER) approach deals with multiple attribute decision analysis (MADA) problem having both qualitative and quantitative attributes under uncertainties and hence facilitates handling of uncertainty in the inference process. Figure 5 shows the system architecture of the influenza suspicion system.

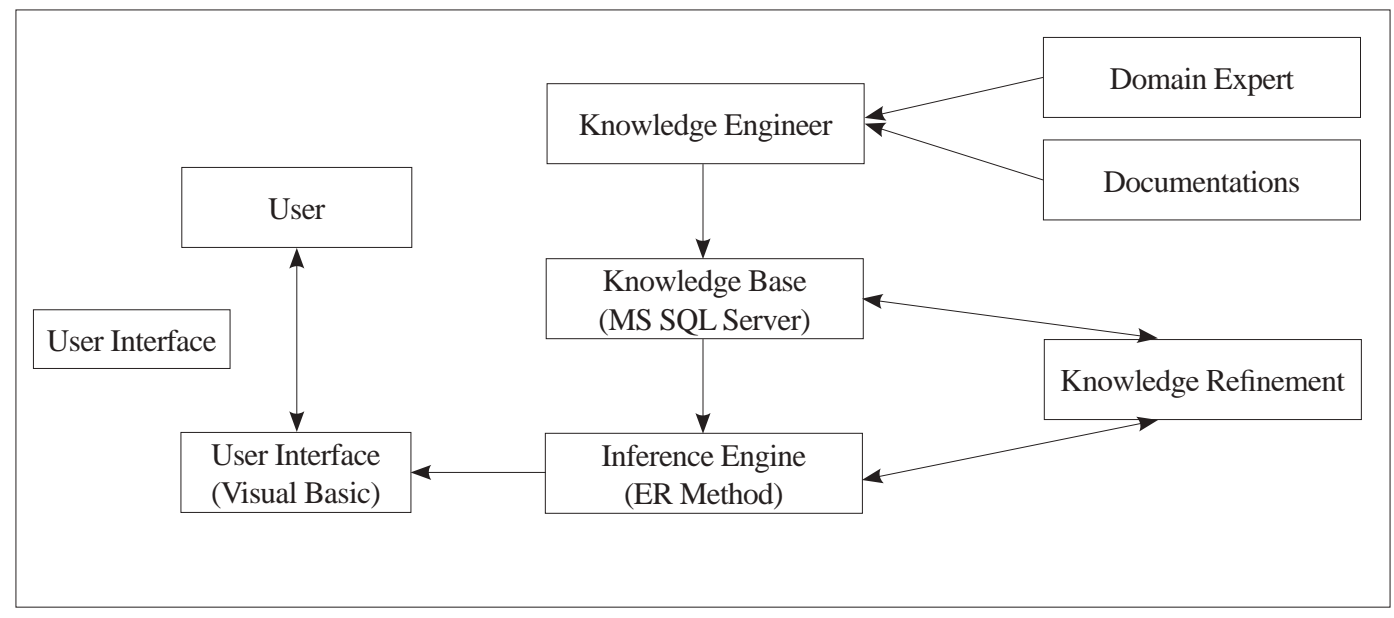

Figure 5: System architecture of the influenza suspicion system

Decision Support System for Malaria and Dengue Disease Diagnosis (DSSMD)

DSSMD was developed by Sharma et al., (2013). Fuzzy logic was used to develop this system. It is used when paramedical human resources need to diagnose the patients, or the patients need to diagnose by themselves as having contracted mosquito-borne diseases. This system can be used anywhere in remote areas especially. DSSMD was developed due to non-availability of pathological and imagingbased medical diagnosis tools in remote areas, many times putting patients' lives in danger which may lead to death. It works by designing a set of rules after identifying the symptoms, and then adding the rules to the database. The rules then are compared with the rules in the database to determine which the correct ones are. The entire symptom is always checked to make sure that the correct symptom matches the correct disease. The rule-base was designed based on the consultation of symptoms with the doctors. Inference then is made by the fuzzy logic toolbox through MATLAB R2013a. The process flow for this system is shown in Figure 7. 


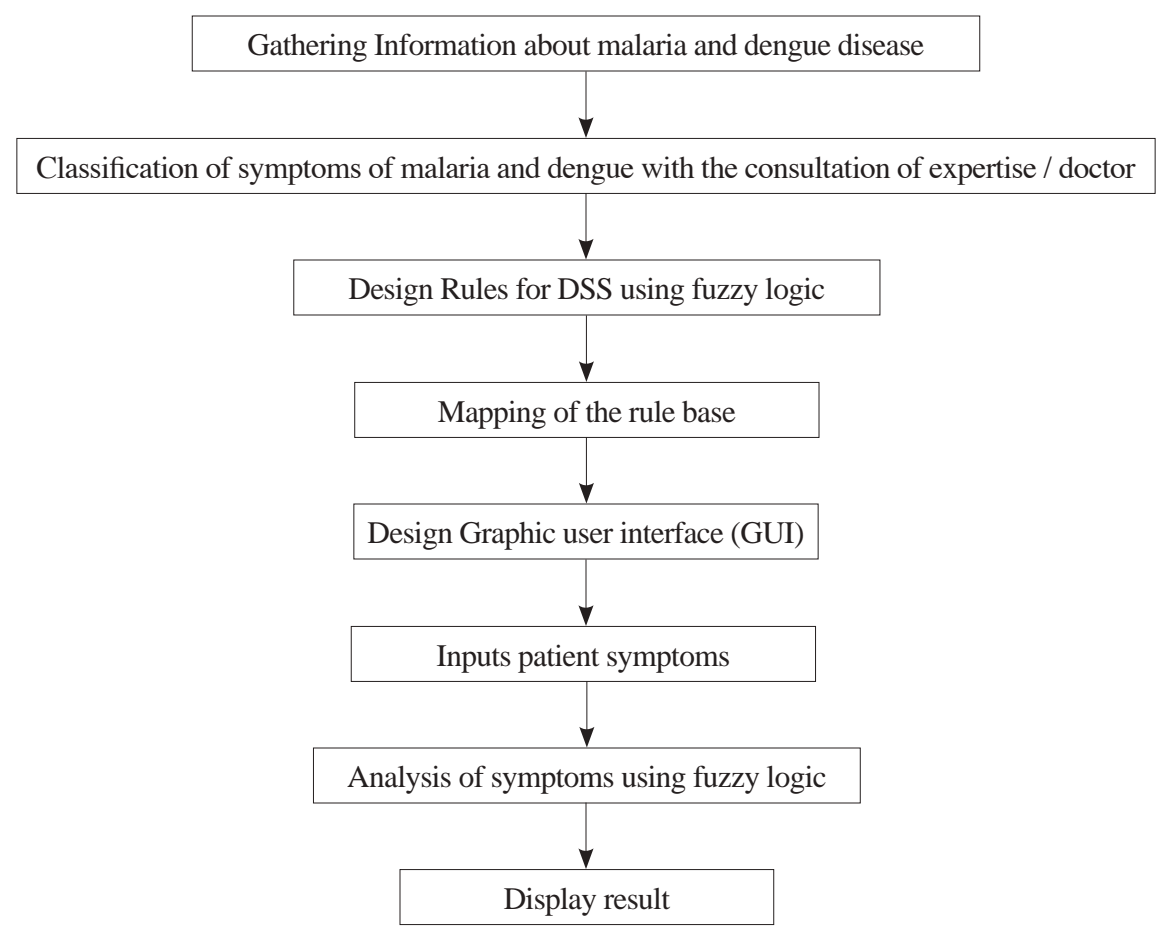

Figure 7: Process flow of DSSMD

\section{Methodology}

This methodology part covers the process of this research and the framework created. It also includes the project planning so that all the work will be done according to the plan. This methodology is a crucial part where it determines what work needs to be done first with the correct implementation or flow of the project. Figure 8 illustrates the framework for this Expert System to Diagnose Dengue Fever.

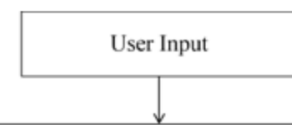

Processing Risk
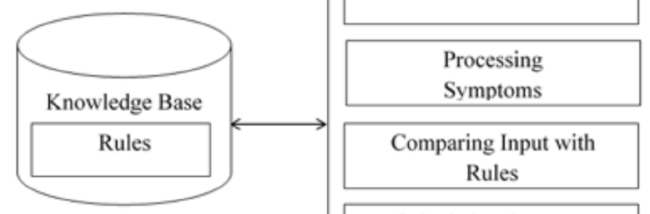

Comparing Input with Rules

Calculating Outcome Percentage

Create Inference
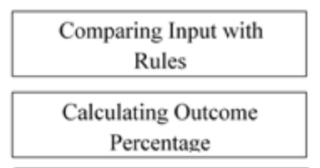

Suspect

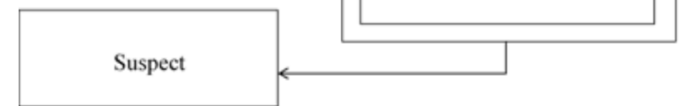

Figure 8: Framework of Expert System to Diagnose Dengue Fever 
At the user input stage, the system provides a web-based graphical interface where users need to input the symptoms they have. The system supports using two browsers, namely Firefox and Seamonkey. In each page, users will answer a question related to the dengue symptoms. Another stage in this system is knowledge base. Knowledge base is identified as a place that stores all the information related to the symptoms that is collected from experts and books. In addition, the knowledge also comes from journals which are documented. In knowledge base, there are rules. Rules are created and designed based on experts' consultation. The symptoms of the patient are keyed into the system and based on the symptoms stored in the knowledge base, the symptoms are processed. Table 1 shows the rules to be followed in knowledge base.

Table 1: Rule 1 in Knowledge Base

\begin{tabular}{cc}
\hline IF & THEN \\
\hline Fever $\left(>40^{\circ} \mathrm{C}\right)$ & $\begin{array}{c}\text { Check other } \\
\text { symptoms }\end{array}$ \\
Fever $\left(>40^{\circ} \mathrm{C}\right)$ with one & $\begin{array}{c}\text { Might not be dengue } \\
\text { symptom }\end{array}$ \\
Fever $\left(>40^{\circ} \mathrm{C}\right)$ with two & Suspected dengue \\
symptoms & Not dengue \\
No Fever & Might be dengue \\
Fever $\left(<40^{\circ} \mathrm{C}\right)$ with two & \\
Fever $\left(<40^{\circ} \mathrm{C}\right)$ with one & Might not be dengue \\
symptom & \\
\hline
\end{tabular}

Figure 9 shows the flow of this system. Processing risk phase requires input from the user regarding the age of the user and other miscellaneous questions to determine whether the user can be declared as having dengue fever or not. Risk processing will process the person's age to determine whether the user is adult or teen and process miscellaneous questions and provide certain percentage based on it.

Symptoms will be processed by asking the user during processing system phase several set of questions on the symptoms of dengue they have. The input keyed in is then processed thoroughly before it is passed to the next process, which is comparing the input with rules in knowledge base. Comparing Input with Rules phase is where input is compared by users with rules in knowledge base. Every single symptom input will be processed and compared to give an outcome to the user.

Calculating Outcome Percentage phase is where the system will compare each input from user and determine its percentage by rules in knowledge base. Percentage is collected and the system will create an inference that will show how bad user is affected by dengue. Percentage of the outcome from the input will not be displayed. Table 2 illustrates the symptoms with conditions and percentages. Inference is made during create inference phase after comparison of the input with the rules is done and outcome percentage is calculated. Decrement or increment of percentage is also set based on the rules. The percentage is then converted into a proper way to describe whether the user is affected by dengue or not.

Lastly, Suspicion of dengue is determined at suspect phase by the inference made by the inference engine. The suspicion of dengue is divided into different expert opinions, namely "Not Dengue", "Might be Dengue", "Might not be Dengue" and "Suspected Dengue". 


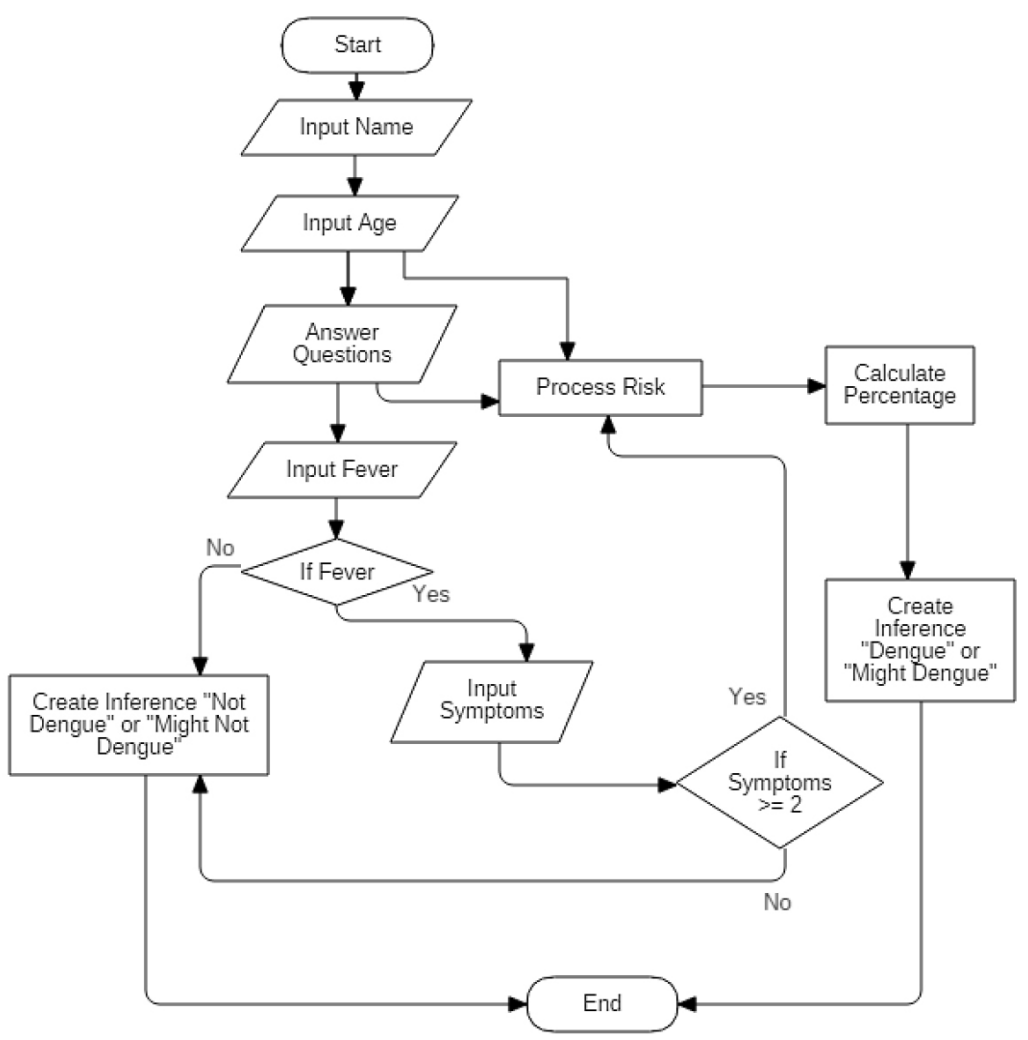

Figure 9: Flowchart of Expert System to Diagnose Dengue Fever

Table 2: Symptoms with conditions and percentages

\begin{tabular}{ccc}
\hline Symptom & Condition & Percentage $(\%)$ \\
\hline \multirow{2}{*}{ Fever } & High & 8 \\
& Mild & 6 \\
& No & 0 \\
Headache & Severe & 8 \\
& Mild & 6 \\
& No & 0 \\
Vomit & Persistent & 8 \\
& Mild & 6 \\
& No & 0 \\
Abdominal & Severe & 8 \\
& Mild & 6 \\
& No & 0 \\
Eye & Severe & 8 \\
& Mild & 6
\end{tabular}




\begin{tabular}{ccc}
\multirow{3}{*}{ Skin } & Yes & 8 \\
& No & 0 \\
\multirow{3}{*}{ Joint } & Severe & 8 \\
& Mild & 6 \\
& No & 0 \\
Muscle & Severe & 8 \\
& Mild & 6 \\
\multirow{3}{*}{ Breath } & No & 0 \\
& Difficult & 8 \\
Bleed & Normal & 0 \\
& Severe & 8 \\
Drowsy & Mild & 6 \\
& No & 0 \\
& Yes & 8 \\
\end{tabular}

\section{Discussion}

Based on Figure 10, there is an increment of dengue cases in Malaysia from 1995 to 2017.

This expert system has provided a solution to diagnose dengue fever at an early stage through the detection of dengue fever by symptoms.

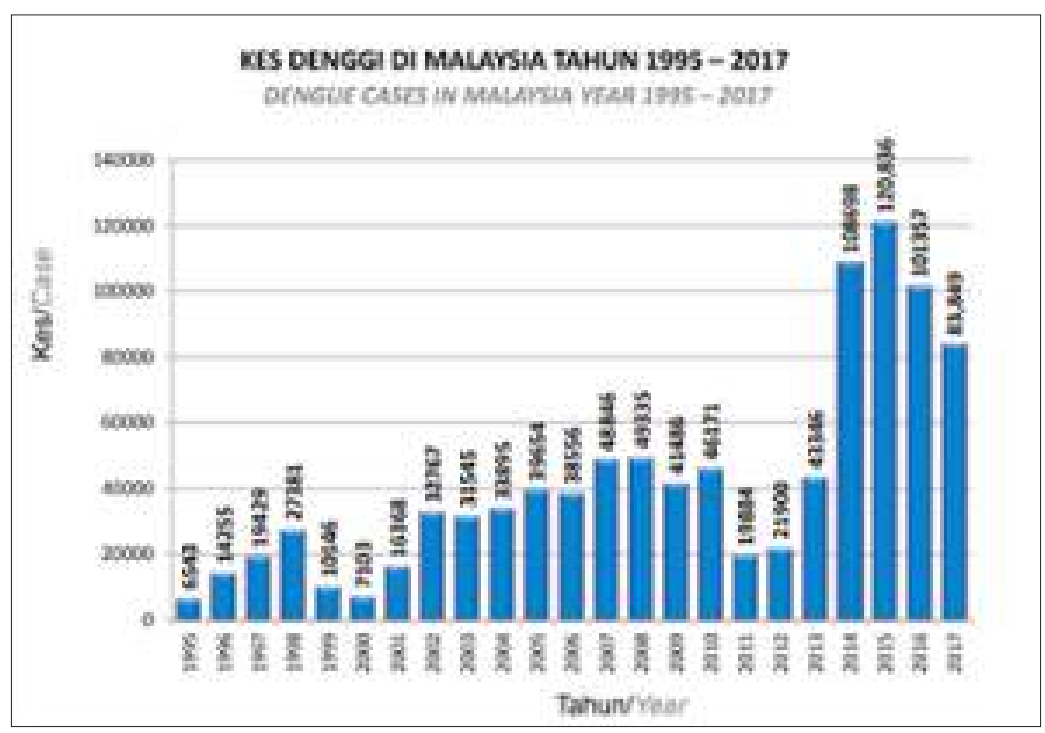

Figure 10: Dengue Cases in Malaysia Year 1995-2017, 2018

Expert System to Diagnose Dengue Fever is an interactive web-based expert system which asks questions regarding dengue fever symptoms. Besides that, it can be accessed anywhere using desktops or laptops. The system is an alternative way, instead of waiting to see a medical doctor, to know whether user has contracted dengue fever or not. Expert System 
to Diagnose Dengue Fever has implemented an artificial intelligence using PROLOG, and it has been integrated with web based application to enable the user to experience similar situation as meeting an expert when using the system. The system may also create awareness of the dengue fever.

The system requires an internet connection to be used due to its platform being web-based. The system does not provide a function to print out the results to be shown to the medical doctor. User of the system needs to have knowledge of the English language. The system needs to be improved in the future. It should be developed for mobile-based platform. The system also should create a print function to prints the results, so that patients can show them to the doctor. Lastly, the expert system can be set up and used for other medical problems. As a conclusion, the system may increase awareness of the user towards dengue fever, which may prompt the user with dengue fever to obtain early treatment, thereby lowering the rates of death due to dengue fever.

\section{Results}

Expert System to Diagnose Dengue Fever provides an output based on symptoms input by user through interactive webpages, and this output may be any of these: Suspected Dengue, Might be Dengue, Might Not be Dengue, and Not Dengue. Expert System to Diagnose Dengue Fever has been evaluated by medical doctors. The system is considered to have served its purpose, which is to detect dengue fever at an early stage through identification of symptoms.

\section{Conclusion}

As a conclusion, Expert System to Diagnose Dengue Fever is a convenient system that can be accessed anywhere and anytime to diagnose dengue fever through symptoms. The system is capable of diagnosing dengue fever like a medical doctor due to the AI implemented in the system. The system lets people diagnose dengue fever by themselves without having to consult a medical doctor, which will reduce the number of people visiting the clinic or the hospital, making the places less crowded.

\section{References}

Bilik Gerakan Denggi Kebangsaan CPRC, Kementerian Kesihatan Malaysia (KKM). (2017). Retrieved from http://idengue. remotesensing.gov.my/idengue/

Dengue and Severe Dengue. (April 2017). Retrieved from http://www.who.int/ mediacentre/factsheets/fs117/en/

History of Dengue. (n.d.). Retrieved from http:// www.denguevirusnet.com/history-ofdengue.html.

Hossain, M. S., Khalid, M. S., Akter, S., \& Dey, S. (2014). A Belief Rule-Based Expert System to Diagnose Influenza.

Kes Denggi di Malaysia Tahun 1995 - 2017. (2018). Retrieved from http://idengue. remotesensing.gov.my/idengue/content/ statistik.pdf

Raúl, B. R., Rocío, M. A., Carlos, G. S., \& Adauto, C. F., (2015). An Expert System Oriented towards the Detection of Influenza and Dengue Developed on Mobile Platforms (BioDnX).

Sharma, P., DBV, S., Manoj, K. B., \& Nidhi, M. (2013). Decision Support System for Malaria and Dengue Disease Diagnosis (DSSMD).

Santosh, K. P., Dipti, P. S., \& Indrajit, M., (2010). An Expert System for Diagnosis of Human Diseases, International Journal of Computer Applications, 1(13).

Sharifah Hanis, Syed Ahmad (2012) An expert system to track dengue fever. Faculty of Computer System \& Software Engineering, Universiti Malaysia Pahang.

Tarig, F., Mohd, N. T., \& Fatimah, I. (2012). Adaptive Neuro-Fuzzy Inference System for diagnosis risk in dengue patient. 